\title{
Article
}

\section{Omnidispersible Microscale Colloids with Nanoscale Polymeric Spikes}

\author{
Douglas G. Montjoy, Harrison Hou, Joong Hwan Bahng, and Nicholas A. Kotov
}

Chem. Mater., Just Accepted Manuscript • DOI: 10.1021/acs.chemmater.0c02472 • Publication Date (Web): 14 Sep 2020

Downloaded from pubs.acs.org on September 15, 2020

\section{Just Accepted}

"Just Accepted" manuscripts have been peer-reviewed and accepted for publication. They are posted online prior to technical editing, formatting for publication and author proofing. The American Chemical Society provides "Just Accepted" as a service to the research community to expedite the dissemination of scientific material as soon as possible after acceptance. "Just Accepted" manuscripts appear in full in PDF format accompanied by an HTML abstract. "Just Accepted" manuscripts have been fully peer reviewed, but should not be considered the official version of record. They are citable by the Digital Object Identifier (DOI®). "Just Accepted" is an optional service offered to authors. Therefore, the "Just Accepted" Web site may not include all articles that will be published in the journal. After a manuscript is technically edited and formatted, it will be removed from the "Just Accepted" Web site and published as an ASAP article. Note that technical editing may introduce minor changes to the manuscript text and/or graphics which could affect content, and all legal disclaimers and ethical guidelines that apply to the journal pertain. ACS cannot be held responsible for errors or consequences arising from the use of information contained in these "Just Accepted" manuscripts. 


\title{
Omnidispersible Microscale Colloids with Nanoscale Polymeric Spikes
}

\author{
Douglas G. Montjoy', Harrison Hou', Joong Hwan Bahng², Nicholas A. Kotov,3,4,5
}

${ }^{1}$ Department of Chemical Engineering, 32Department of Biomedical Engineering, ${ }^{4}$ Department of Materials Science, and ${ }^{5}$ Biointerfaces Institute, University of Michigan, Ann Arbor, Michigan 48109, United States

${ }^{2}$ Department of Electrical Engineering, California Institute of Technology, Pasadena, California 91125, USA

ABSTRACT: Particle stability in a multiplicity of fluid environments is critical for colloids used in catalysis, sensing, and composites. Hedgehog particles (HPs), inspired by the spiky topology of pollen grains and viral capsids, enable dispersion stability regardless of whether their polarity matches that of the solvent. Previous implementations of HPs were all based raon rigid spikes from inorganic materials, such as $\mathrm{ZnO}$, whereas polymeric spikes offer a unique spectrum of optical, chemical, thermal, and mechanical properties including potential stimuli-responsive behavior. Microscale particles with nanoscale polymeric spikes referred to here as tendril particles, were made by layer-by-layer assembly of polyallylamine films deposited onto rigid $\mathrm{ZnO}$ templates and then crosslinked with glutaraldehyde. Tunable broadband scattering is observed upon partial removal of the $\mathrm{ZnO}$ with complete removal resulting in semi-rigid hollow polymer sleeves. While being hydrophilic, they disperse in nonpolar media such as heptane and high ionic strength aqueous media. Gradual removal of $\mathrm{ZnO}$ nanorods affords spectral tuning of the near-infrared band associated with light scattering from the high refractive index spikes. The polymer spikes also allow for loading of cargo nanoparticles, molecules, and polymers. By adding poly $(\mathrm{N}$-isopropylacrylamide-coacrylic acid) subunits, controlled aggregation is observed in response to temperature. Structural integration of dopamine moieties into the layered films allows for controlled aggregation in response to alkaline conditions. The mechanical and structural flexibility of tendrils with sleeve-like morphology enables a new generation of multifunctional particles with properties controlled by their nanoscale surface topography.

\section{Introduction}

Surface corrugation with stiff spikes can be used as a universal strategy to reduce van der Waals (vdW) forces between dispersed particles by more than an order of magnitude. ${ }^{1}$ The drastic reduction of attractive forces leads to unexpected colloidal stability of particles with hydrophilic inorganic spikes in hydrophobic solvents and vice versa. ${ }^{2}$ This effect also leads to increased stability of topologically engineered colloids in high ionic strength environments, 3,4 in contrast to the predictions of Derjaguin, Landau, Verwey and Overbeek (DLVO) theory about the colloidal stability of smooth spherical particles. The generality of this effect can be seen in many particles with spiky geometries that were tested for omnidispersibility. Particles with spikes that were conformably coated with organic layer-by-layer assembled (LBL) films ${ }^{5-10}$ maintained dispersion stability verifying the role of particle geometry in the reduction of Tendril Particles

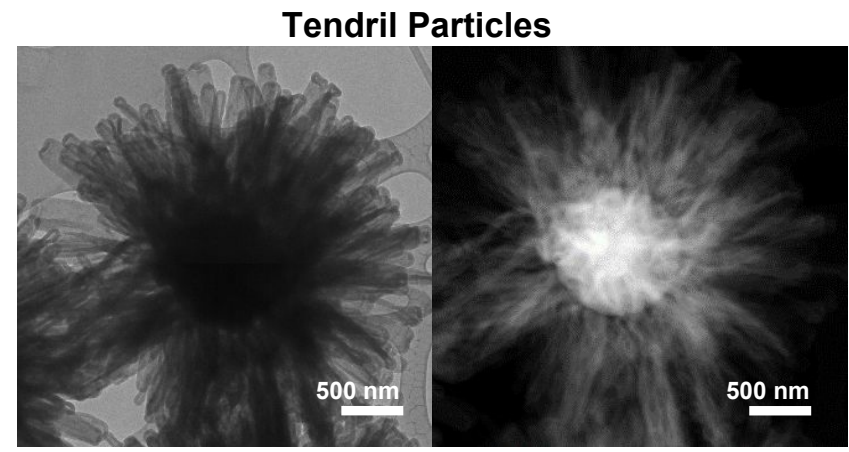

van der Waals forces. ${ }^{3}$ There are multiple other examples of spiky and flower-like particles made from different inorganic materials, ${ }^{11-14}$ which were not tested for dispersibility in 'difficult' solvents, but are expected to display similar colloidal properties. Special functionalities were also found for particles with interfaces decorated by pillars at molecular scale ${ }^{15}$ substantiating the utility of the topographically engineered colloids.

Besides omnidispersibility, rigid inorganic spikes impart hedgehog particles (HPs) with other unique properties, such as a broad band extinction band in the near IR part of the spectrum, ,2,3,16,17 which is attributed to strong light scattering of the radial spikes. ${ }^{2,17}$ Realization of HPs with fully polymeric yet sufficiently rigid spikes would engender these particles with additional functions, exemplified by: (a) tunable extinction bands in the visible spectrum; (b) nanoscale cargo compartments in the spikes; and (c) stimuli-responsive behavior. Note, 
however, that all of these functions originate in the structural reconfigurability of the polymers, and their reversibility should diminish omnidispersibility because the softness of nanoscale polymeric spikes result in recovery of strong van der Waal attraction and other attractive short-range forces.

In this study, we aimed for well defined surface corrugation with polymers which would enable responsive control of dispersion, which has not been achieved in previous works. Polymeric surface corrugation has been introduced previously with nanoparticle coatings, emulsion polymerization, and through nanoprecipitation. ${ }^{1,18,19} \mathrm{HPs}$ with polymeric spikes-or tendril particles (TPs)-can be made using sequentially deposited layers of polyelectrolytes to enable deposition of controlled layer of polymers on highly curved surfaces. ${ }^{8,20-22}$ After removal of the zinc oxide nanorods used as templates, the spikes acquire their sleeve-like morphology. In order to retain omnidispersibility, the constituent polymers of TPs spikes must be cross-linked to acquire rigidity. In addition to enhanced colloidal stability, the polymeric spikes make it possible to vary the optical properties of the particles and load them with nanoscale cargo exemplified by gold nanoparticles (NPs). Through introduction of environmentally responsive molecular subunits, we also show controlled aggregation in response to temperatureand $\mathrm{pH}$ stimuli with both practical and fundamental significance.

\section{Results and Discussion}

Polyelectrolytes and multilayers are an ideal materials platform for engineering TPs because they enable 'soft' materials with high mechanical properties. ${ }^{23-25}$ The topographical engineering of particles requires spikes with high compressive and bending stiffness to diminish $\mathrm{vdW}$ forces between the interacting particles.

Furthermore, polyelectrolytes and multilayers allow for encapsulation of a large variety of functional components that will broaden the spectrum of functionalities of HPs; these components include NPs, ${ }^{26,27}$ enzymes, ${ }^{28,29} \mathrm{DNA},{ }^{30}$ dendrimers, ${ }^{31}$ drugs, ${ }^{32-34}$ dyes, 35 conductive polymers, and other nanoscale components of advanced materials. Moreover, polyelectrolytes are also capable of exhibiting $\mathrm{pH}$-, temperature-, and light-responsive behaviors. ${ }^{27,36,37 .}$

\subsection{Engineering of Tendril Particles. Following}

previous studies of micro- and nanocapsules, $5,20,22,38$ and HP-based biosensors, ${ }^{3}$ polyelectrolyte films on HPs were made by sequential deposition of polyacrylic acid (PAA) and poly(allylamine) hydrochloride (PAH). Young's moduli of PAA/PAH layers vary largely depending on thickness and crosslinking, ranging from $290 \mathrm{MPa}$ when not crosslinked to 1.9 GPa for crosslinked films. This is, of course much smaller that the stiffness of pristine $\mathrm{ZnO}$ nanorods ( $800 \mathrm{GPa}),{ }^{24,39}$ which represents one of the challenges of this study. The constituent components of these multilayers exhibit high glass transition at $128^{\circ} \mathrm{C}$ for PAA and $223^{\circ} \mathrm{C}$ for PAH, which can lead to multilayers ina glassy state at room temperature. ${ }^{\circ}$

Highly uniform and conformal (PAA/PAH) $)_{2}$ coatings of ca. $10 \mathrm{~nm}$ thickness are formed on HPs after two cycles of LBL deposition (Figure $\mathbf{S}_{\mathbf{1}}$ ). TPs are produced when the $\mathrm{ZnO}$ template nanorods are partially or completely dissolved in acidic media. Importantly, the polymeric multilayers were cross-linked with glutaraldehyde to increases the stiffness of the PAA/PAH composites by more than a factor of two, to $680 \mathrm{MPa} .^{24}$ Otherwise, they collapse when the nanorods are dissolved due to low $\mathrm{pH}$ (Figure S2).

Energy dispersive spectroscopy (EDS) was used to examine the $\mathrm{Zn}$ content that remained in the spiky shells of the TPs. A large amount of $\mathrm{Zn}$ ions ( $43 \%$ of $\mathrm{Zn}$ (herein referred to as $43-\mathrm{Zn}$ TPs)) in the form of nanorods remain in the polymeric shell after glutaraldehyde crosslinking in $1 \mathrm{M}$ boric acid for two hours, with the latter assisting $\mathrm{ZnO}$ removal(Figure 1). Additional incubations with o.o1 $\mathrm{M}$ and $0.1 \mathrm{M} \mathrm{HNO}_{3}$ for 72 hours gradually reduced the amount of zinc to $2.9 \%$ (2.9-Zn TPs) and 0.57\% (o.57-Zn $\mathrm{TPs}$ ), respectively, completely removing remaining $\mathrm{ZnO}$ nanostructures and resulting in hollow polymer spikes (Table S1), or tendrils. Scanning transmission electron microscopy-EDS (STEM- EDS) mapping shows that the tendrils consist of primarily carbon, with oxygen nitrogen; a small amount of zinc present as well, most likely due to coordination with LBL films (Figure $\mathbf{S}_{3}$ ). When the crosslinking occurs at an elevated $\mathrm{pH}$, the $\mathrm{ZnO}$ spikes remain intact (Figure $\mathbf{S}_{4}$ ). Similar hollow shell structures are observed when solely $\mathrm{ZnO}$ nanorods are treated with glutaraldehyde at lower $\mathrm{pH}$ (Figure $\mathrm{S}_{\mathbf{5}}$ ). Zinc content representative of the remaining $\mathrm{ZnO}$ nanostructures inside the tendrils has a dramatic effect on the absorbance spectrum of the samples (Figure 1g). As Zncontent becomes smaller, the broad near-infrared scattering band blue shifts, which can be related to the gradual reduction of refractive index of the spikes from $n$ $=1.97(\mathrm{ZnO})$ to $n=1.53$ (characteristic of polymeric multilayers). The finite-difference time-domain (FDTD) simulation of a model HP with gradually diminishing refractive index of the spikes from $n=1.85$ to $n=1.53$ verified this mechanism of spectral changes during $\mathrm{HP} \rightarrow \mathrm{TP}$ transition with near $\mathrm{o} \% \mathrm{ZnO}$ remaining for TPs (Figure 1h)

The number of LBL deposition cycles and dimensions of $\mathrm{ZnO}$ templates are essential for the successful engineering of TPs. The template spikes require a minimal diameter of ca. $75 \mathrm{~nm}$; this was proven after an attempt to form TPs from thin spike HPs (63 $\mathrm{nm}$ diameter) where the tendrils 


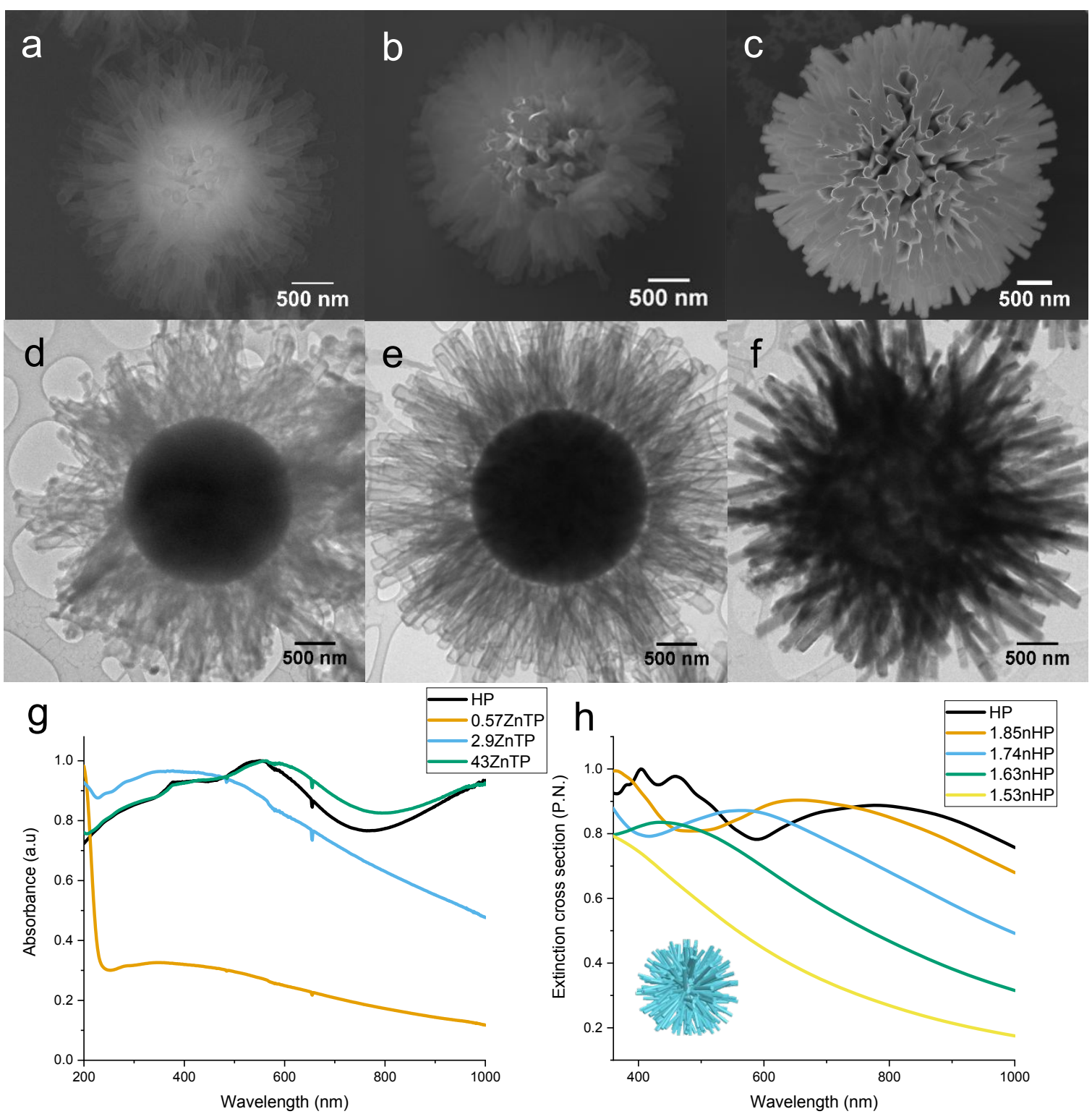

Figure 1. SEM (a,b,c) and TEM (d,e,f) images of two-bilayer TPs with various Zn content, (a,b) o.57-Zn (b,e) 2.9-Zn (c,f) 43-Zn TP. (g) Extinction spectra of TPs with different Zn content. (h) Bottom left: FDTD model HP with spike length of $1463 \mathrm{~nm}$, spike thickness of $163 \mathrm{~nm}$, and 500 spikes; Normalized calculated extinction cross section of model HP with different refractive indices (nHP), corresponding to $75 \%(1.85 \mathrm{nHP}), 50 \%(1.74 \mathrm{nHP}), 25 \%(1.63 \mathrm{nHP})$, and $0 \%(1.53 \mathrm{nHP})$ percentages of $\mathrm{ZnO}$ remaining. The full model parameters and are listed in the methods. ${ }^{[23,32,33}$

collapsed after the $\mathrm{ZnO}$ extraction (Figure S6). If too many LBL deposition cycles are performed, a polymer web is formed over the spikes, mitigating corrugation and increasing vdW attractions (Figure S6). Varying the length of the spikes on HPs allows a similar degree of freedom for TP structure formation. Utilizing $1 \mathrm{M}$ boric acid in the step to wash away $\mathrm{ZnO}$, allows for very uniform and robust sleeves of varied diameters and lengths ranging from greater than $500 \mathrm{~nm}$ to slightly less than $2000 \mathrm{~nm}$ (Figure 2). When dissolving HPs with larger $\mathrm{ZnO}$ nanorods, longer incubation times with acid are needed to ensure their full dissolution (Figure $\mathbf{S}_{7}$ ).In addition, TPs based on polydisperse HPs from small 296 $\mathrm{nm} \mathrm{SiO}{ }_{2}$ cores can also be synthesized (Figures 2c,f,S8). As seen with larger cores, spikes that were thin and short did not form stable TPs while larger spikes form TPs with distinct spiky morphology (Figure s9). We expect that.forming TPs with cores smaller than $300 \mathrm{~nm}$ would not allow for sufficient width for $\mathrm{ZnO}$ nanorod growth. As a demonstration of the generality of this method, we also made TPs using ethylcarbodiimide (EDC) as a crosslinking agent instead of glutaraldehyde. However, this type of TP is only stable, with thicker

(PAA/PAH) ${ }_{3}$ PAA bilayer films and shows a more rough and less uniform morphology than the ones cross-linked with glutaraldehyde (Figure S1o).

2.2 Dispersions of Tendril Particles. In a previous study, we determined that when surface corrugation is preserved with the addition of polymer and nanoparticles 


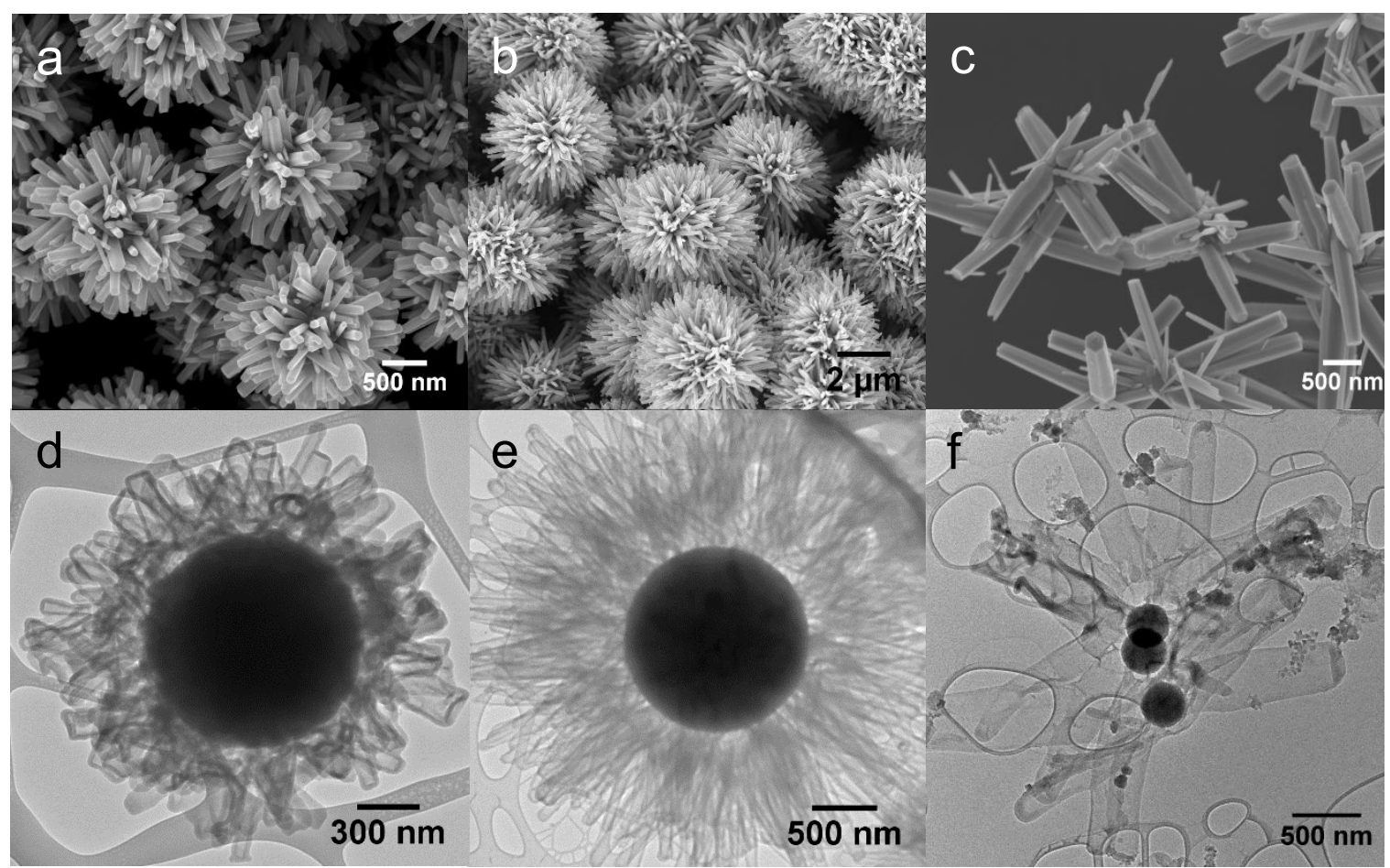

Figure 2. (a,b.c) SEM and (d,e,f)TEM images of (a,b) HPs with $1.1 \mu \mathrm{m}$ average diameter $\mathrm{SiO}_{2}$ cores with average spike lengths of $540 \mathrm{~nm}$ and $1930 \mathrm{~nm}$ and average spike widths of $99 \mathrm{~nm}$ and $146 \mathrm{~nm}$ respectively;(c) HPs with $296 \mathrm{~nm}$ average diameter $\mathrm{SiO}_{2}$ cores with an average spike length of $1253 \mathrm{~nm}$ and average spike width of $188 \mathrm{~nm}$; (d,e,f) TPs prepared from HPs in (a), (b), (c) respectively after glutaraldehyde crosslinking and 72 hour treatment with $1 \mathrm{M}$ boric acid.

omnidispersibility of HPs is maintained. 3 Flexible spikes in TPs that are deformable result in softer interaction potentials. We observed that the TPs maintain dispersibility in nonpolar andhigh ionic strength environments (Figure 3, S11-S12), indicating that the stiff crosslinked polymer enables reduction of van der Waals forces despite a Young's modulus of the tendrils that is about three orders of magnitude smaller than that of $\mathrm{ZnO}$. Confocal microscopy and SEM images confirm well-dispersed particles in highly nonpolar heptane and in $1 \mathrm{M} \mathrm{NaCl}$ (Figure 3, S12). It would be expected that the polymer would have reduced attractive van der Waals forces, however, only reduction of contact area consistent with rigid spikes enables dispersion in a nonpolar environment. ${ }^{4}$ Based on these results, we believe that the cross-linked polymer is semi-rigid and does not completely deform upon contact, allowing for a marked reduction of van der Waals forces. Stability in high ionic strength environments as well as nonpolar environments.has broad importance in biosensing, catalysis, and other fields.

2.3 Loading of the Sleeve-like Compartments. To demonstrate the conceptual possibility of loading of cargo in TPs, we first loaded Au NPs using LBL deposition ${ }^{[3]}$ including them in LBL sequence PSS/PDDA/AuNP (AuTP) and PSS/PDDA (o.5 M NaCl)/AuNP (AuSTP). With tendril shells formed around the NPs, we are able to crosslink Au NPs into the tendril structure with controlled loading (Figure 4a-d, S13). We see a large broadening of the optical absorbance in the visible range of these TPs as a function of the density of the Au NP coating (Figure $\mathbf{4 h}, \mathbf{S} \mathbf{3}$ ). Additionally, small nanoparticles can be loaded in an analogous manner to obtain visible light-responsiveness. ${ }^{41}$

To evaluate TPs forthe loading of macromolecules, we used fluorescein isothiocyanate-dextran (FITC-dextran). In this case, we were able to make the polyelectrolyte film permeable at $\mathrm{pH} 3$ and impermeable at $\mathrm{pH} 10 .{ }^{42-44}$ The acidity-dependent phase transition in polyelectrolyte multilayers allows for encapsulation of FITC-dextran into the hollow spikes by loading and then subsequent raising of the $\mathrm{pH}$ (Figure $\mathbf{4} \mathbf{e}-\mathbf{g})$.

Encapsulation was also demonstrated with hydrophilic (rhodamine B) and hydrophobic (nile red) small molecules (Figure $\mathrm{S}_{14}$ ) Loading is achieved at $\mathrm{pH}_{3}$ and with ethanol respectively. For Nile Red dye, the encapsulation is confirmed by the strong fluorescence maintained in aqueous solutions at $\mathrm{pH}_{3}$ or $\mathrm{pH} \mathrm{H}_{1}$, even though the molecule is unstable in water.

With small molecules, release is different, as they are able to diffuse inside regardless of $\mathrm{pH}$ as can be seen with Rhodamine $\mathrm{B}$ at $\mathrm{pH} 10.45$ In this case, combination with another release mechanism such as encapsulated gold nanoparticles (Figure 4a-d) could enable light-based release in combination with laser pulses as shown in previous studies. ${ }^{41}$ 


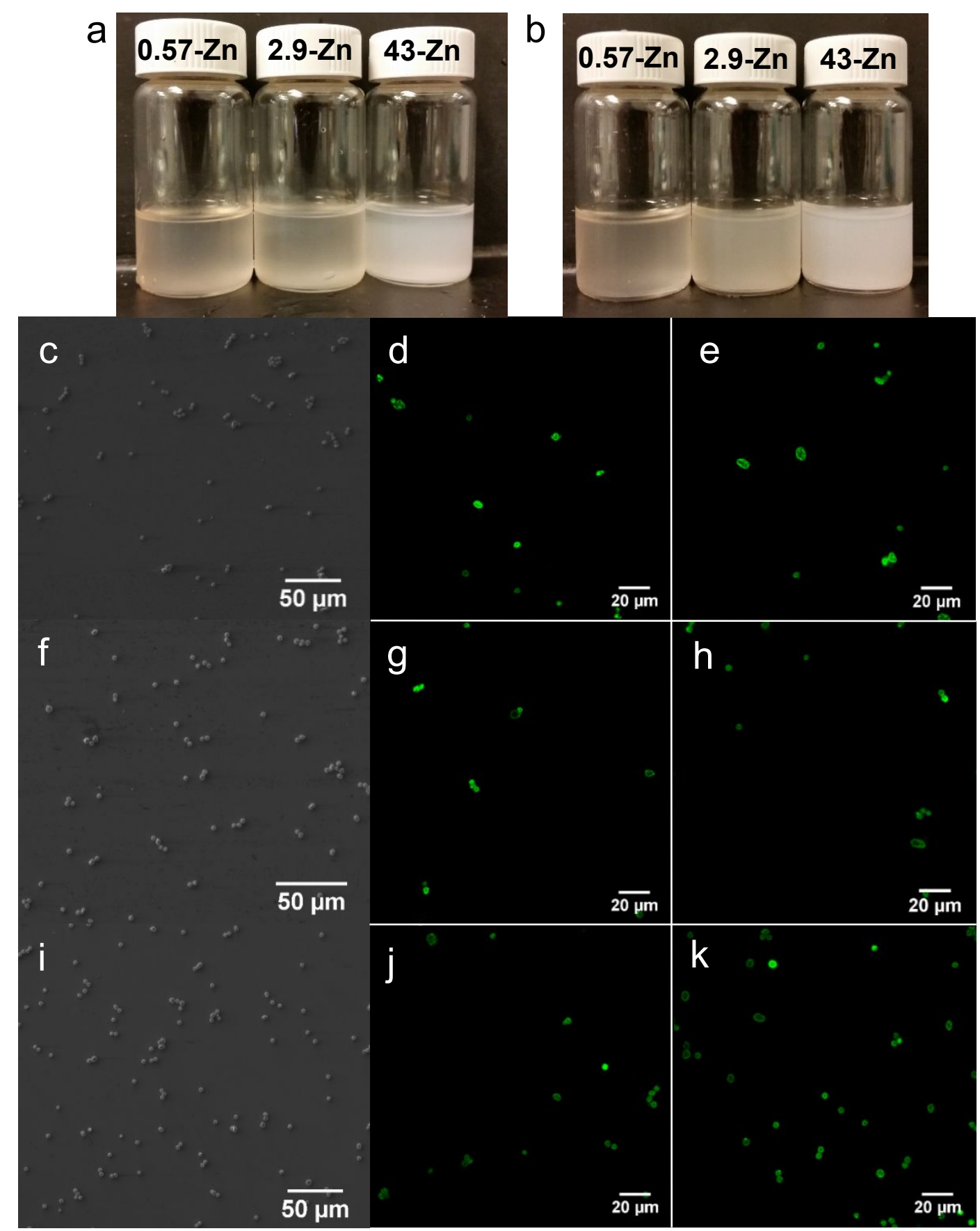

Figure 3. Photographs of TP dispersions $(0.5 \mathrm{mg} / \mathrm{mL})$ in heptane (a) and $1 \mathrm{M} \mathrm{NaCl}$ in water (b) with increasing zinc content from left to right. SEM (c,f,i) and confocal images $(\mathbf{d}, \mathbf{e}, \mathbf{g}, \mathbf{h}, \mathbf{j}, \mathbf{k})$ of $0.57-\mathrm{Zn}$ TPs in heptane $(\mathbf{c}, \mathbf{d})$ and $1 \mathrm{M} \mathrm{NaCl}(\mathbf{e}), 2.9$-Zn TPs in heptane $(\mathbf{f}, \mathbf{g})$ and $1 \mathrm{M} \mathrm{NaCl}(\mathbf{h})$, and $43-\mathrm{Zn}$ TPs in heptane $(\mathbf{i}, \mathbf{j})$ and $1 \mathrm{M} \mathrm{NaCl}(\mathbf{k})$. All SEM and confocal samples were prepared at $0.1 \mathrm{mg} / \mathrm{mL}$

With the capability of selective loading and release by $\mathrm{pH}$, these TPs could have industrial uses, such as incorporating anti-corrosion coatings or for encapsulation of therapeutics. ${ }^{46,47}$ In addition, through the creation of hollow polyelectrolyte cores in addition to spikes, TPs would enable high loadings or selective loadings in each compartment.

\subsection{Temperature and $\mathrm{pH}$-stimulated Agglomeration} of Tendril Particles. The ability to modulate a polymer's reversible thermal response has been well-explored with polymers such as poly( $\mathrm{N}$-isopropylacrylamide) (PNIPAM). ${ }^{48-50}$ In addition, $\mathrm{pH}$-responsive behavior can be utilized for many different biological applications and can be achieved with different polymeric subunits including dopamine. ${ }^{51}$ Introduction of thermoresponsive and $\mathrm{pH}$-responsive polymers to TPs is expected to result in the loss of dispersion stability depending on media conditions. To test thermoresponsive capability, we used LBL to coat TPs with films of poly(N-isopropylacrylamide-coacrylic acid) (PNIPAM TPs) and evaluated particle stability at different temperatures. Particle stability was evaluated at 

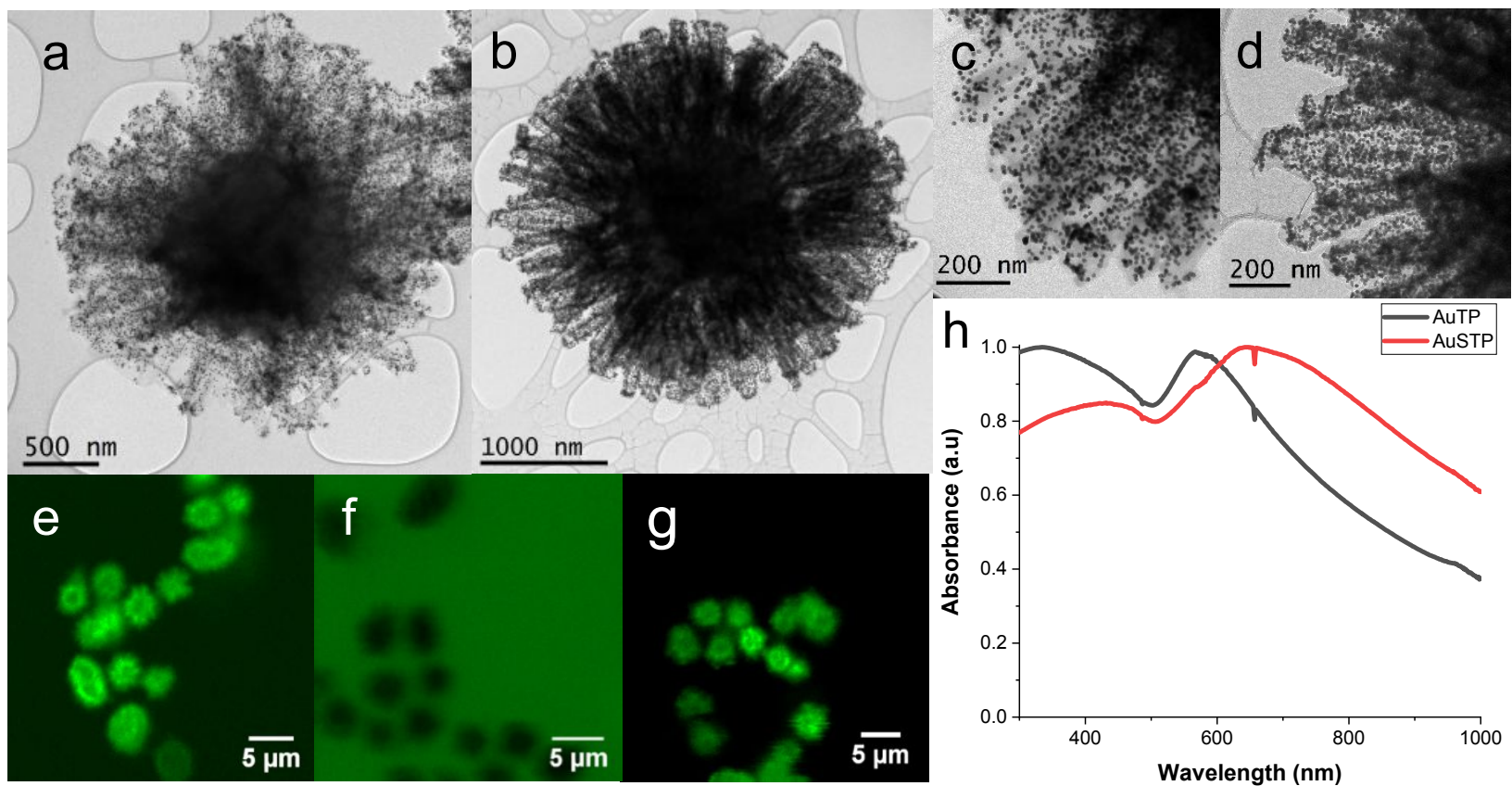

Figure 4. TEM of tendril particles with PSS/PDDA/AuNP (AuTP) (a,c) and with PSS/PDDA (o.5 M NaCl)/AuNP (AuSTP) (b,d) layers encapsulated. Confocal microscopy images of TPs dispersed in $1 \mathrm{mM}$ FITC-Dextran (40oo $\mathrm{MW} ; 1 \mathrm{mM})$ at $\mathrm{pH} 3(\mathbf{e})$ and $\mathrm{pH}$ 10 (f). Encapsulation of FITC-dextran by raising $\mathrm{pH}$ after treatment at $\mathrm{pH} 3$ and washing off excess FITC-dextran (g). Normalized absorbance spectrum for AuTP and AuSTP (h).

room temperature, $40^{\circ} \mathrm{C}, 60^{\circ} \mathrm{C}$, and $80^{\circ} \mathrm{C}$. PNIPAM TPs rapidly sediment within five minutes after being incubated at $60{ }^{\circ} \mathrm{C}$ and $80^{\circ} \mathrm{C}$ while normal PAA TPs show limited sedimentation at these temperatures (Figure $\mathbf{5}^{\mathbf{a}-\mathbf{d}, \mathbf{S 1 5}}, \mathbf{S 1 6}$ ). Some aggregation is observed at $40{ }^{\circ} \mathrm{C}$ and very little sedimentation at room temperature (Figure $\left.\mathbf{S 1 7}_{\mathbf{1 7}}, \mathbf{S 1 8}\right)$. Normal, non-modified TPs (PAA TPs) show some thermoresponsive behavior, likely due to disruption of hydrogen bonding, but exhibit less aggregation than the PNIPAM TPs.We believe PNIPAM collapses at high temperature and covers the TP, resulting in hydrophobicinteractions and aggregation. The transformation of PNIPAM has been shown to be reversible,,$^{52}$ allowing for recovery and reuse of the TP.

Dopamine was conjugated to PAH using glutaraldehyde to create $\mathrm{pH}$-responsive TPs (DOP TPs) (Figure S19). Using confocal microscopy, limited aggregates are observed at $\mathrm{pH}$ 5.6, compared to large aggregates at $\mathrm{pH}$ 7.4. (Figure $5 \mathbf{e - f}$ ). Additionally, absorbance was measured over 30 minutes for DOP TPs at $\mathrm{pH} 5.6$ and $\mathrm{pH} 7.4$ in o.01 M phosphate buffer saline (PBS) to analyze sedimentation. There was a decrease in absorbance (at a wavelength of $275 \mathrm{~nm}$ ) of almost $50 \%$ at $\mathrm{pH} 7.4$ while only $20 \%$ at $\mathrm{pH} 5.6$ over 30 minutes (Figure S2o). When PAA TPs are tested, little difference is observed between the two $\mathrm{pH}$ values indicating that the dopamine group is responsible for the change (Figure S21). Dopamine contains catechol groups, that, when exposed to alkaline conditions, oxidize to quinone or semiquinone forms, which are reactive and result in covalent crosslinking. 53,54 In this case, the reaction is irreversible unlike the NIPAM TPs, which we observe when analyzing sedimentation of recovered DOP TPs after the $\mathrm{pH}$ is readjusted to 5.6 from 7.4 (Figure S22). Irreversible aggregation of $\mathrm{pH}$ - and temperature-responsive TPs under conditions when the polymeric spikes become soft and reconfigurable confirms the fundamental mechanisms of omnidispersibility in spiky particles related to reduction of close-range attractive interactions.

\section{Conclusions}

Formation of highly corrugated colloids with polymeric stiff spikes is possible when polyelectrolyte multilayers are crosslinked on a $\mathrm{ZnO}$ template. After removal of $\mathrm{ZnO}$, TPs maintain excellent dispersion stability in nonpolar and high ionic strength media. Gradual removal of $\mathrm{ZnO}$ nanorods affords spectral tuning of the near-infrared band associated with light scattering from the high refractive index spiky shell. The mechanical and structural flexibility of tendrils with sleeve-like morphology enables environmentally responsive TPs. Their responsiveness to thermal and $\mathrm{pH}$ stimuli directly confirms the physicochemical mechanism of their enhanced dispersibility related to the drastic reduction of van der Waals forces in particles with stiff nanoscale spikes.

\section{Experimental Section}

4.1 Materials: All materials were purchased from commercial sources: poly(diallyldimethylammonium chloride) (PDDA), sodium polystyrenesulfonate (PSS), polyacrylic acid (PAA), poly(allylamine hydrochloride) (PAH), zinc nitrate hexahydrate $\left(\mathrm{Zn}\left(\mathrm{NO}_{3}\right)_{2} \cdot 6 \mathrm{H}_{2} \mathrm{O}, \geq 99 \%\right.$ purum), hexamethylene tetramine $\left(\mathrm{C}_{6} \mathrm{H}_{12} \mathrm{~N}_{4}, \geq 99.5 \%\right.$ puriss $)$, boric acid, ammonium hydroxide ( $28 \mathrm{wt} \%$ solution), tetraethyl orthosilicate (TEOS), potassium chloride, glutaraldehyde ( $25 \mathrm{wt} \%$ solution), and zinc oxide NPs (50 wt $\%$ solution) were purchased from Sigma-Aldrich (St. Louis, $\mathrm{MO}$ ); grids with ultrathin carbon film on holey carbon film support for transmission electron microscopy (TEM) 


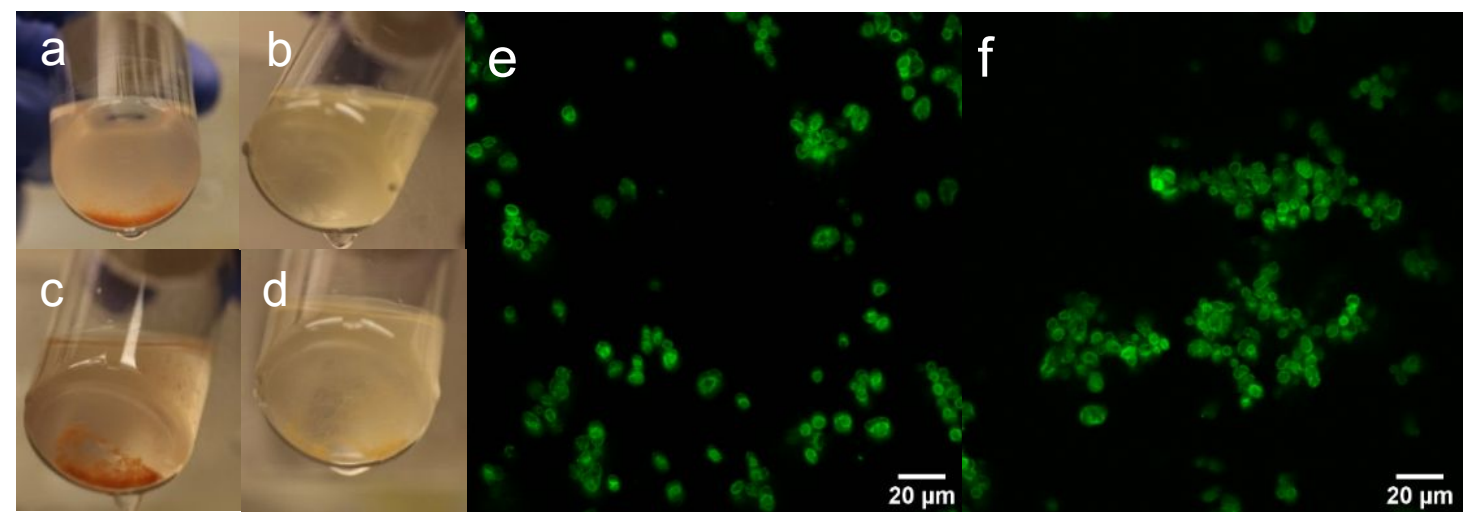

Figure 5. Images of dispersions (0.5 mg/mL) of poly(N-isopropyl acrylamide tendril particles) (PNIPAMTP)(a,c) and 2.9-ZnTP (b,d) after 5 minutes in water bath at $(\mathbf{a}, \mathbf{b}) 60^{\circ} \mathrm{C}(\mathbf{c}, \mathbf{d}) 80^{\circ} \mathrm{C}$. Confocal microscopy of dopamine-modified TP (DOPTP) (o.1 $\mathrm{mg} / \mathrm{mL}$ ) at $\mathrm{pH} 5.6(\mathbf{e})$ and $\mathrm{pH} 7.4(\mathbf{f})$ in $0.01 \mathrm{M}$ PBS.

measurements and silicon wafers for scanning electron microscopy (SEM) measurements from Ted Pella (Redding, CA). Carboxylated polystyrene (PS) was ordered from Polysciences, Inc. Polycarbonate track-etched membranes (o.8 micron, $90 \mathrm{~mm}$ ) were ordered from Sterlitech. All chemicals were ordered from Sigma-Aldrich unless noted otherwise.

\subsection{Synthesis and Layer-by-Layer Coating of Core}

Particles. Silica microparticles were produced by a modified Stöber process. ${ }^{55,56}$ Briefly, $0.017 \mathrm{~g}$ of potassium chloride, 6.75 $\mathrm{mL}$ water, and $9 \mathrm{~mL}$ ammonium hydroxide were added to 65 $\mathrm{mL}$ ethanol. A solution of $34 \mathrm{~mL}$ ethanol and $2.2 \mathrm{~mL}$ of TEOS were added at a rate of $30 \mathrm{~mL} / \mathrm{h}$ via syringe pump under vigorous stirring. Silica cores were coated with poly(diallyldimethyl ammonium chloride) polystyrene sulfonate and in a layer-by-layer process as previously mentioned. Briefly, $5 \mathrm{~mL}$ core solution $(20 \mathrm{mg} / \mathrm{mL})$ was mixed with $25 \mathrm{~mL}$ polyelectrolyte solution $(1 \mathrm{mg} / \mathrm{mL}, 1 \mathrm{M} \mathrm{NaCl})$ and incubated for $20 \mathrm{~min}$. Cores were then centrifuged at 4000 RPM for 10 min and washed with water 3 times before repeating the process with the next layer. $296 \mathrm{~nm} \mathrm{\textrm {SiO } _ { 2 }}$ beads were created using a modified stöber method. A TEOS concentration of $0.28 \mathrm{M}$, Water concentration of $6 \mathrm{M}$, and ammonium hydroxide $.56 \mathrm{M}$ were used in ethanol. TEOS was added after ethanol, ammonium hydroxide, and water were mixed for 10 minutes and particles were stirred overnight. 57

4.3 Synthesis of Hedgehog Particles. Polystyrene and $\mathrm{SiO}_{2}$ core HPs were synthesized using a scaled sonothermal synthesis based on that of Bahng et al. A $5 \mathrm{~mL}$ aliquot of coated core solution (2.5 wt $\%$ ) was mixed with $160 \mathrm{~mL} \mathrm{ZnO}$ seed solution (0.025 wt\%) and incubated for 1 h. 4 Particles were then filtered using a 0.8 micron track etch membrane and combined with equimolar $(25 \mathrm{mM})$ solutions of zinc nitrate hexahydrate $(\mathrm{ZnH})$ and hexamethylenetetramine (HMT) in water. Concentrations of the precursors were varied to tune the width of the spikes; the default concentration was $25 \mathrm{mM}$ with 2 sonications. The solution was then diluted to a final volume of $1.6 \mathrm{~L}$ and sonicated using a Hielscher 10ooUIP HdT sonicator for $1.5 \mathrm{~h}$. Samples were purified by removing excess zinc oxide nanorods after sedimentation of HPs. Longer HPs were created by performing additional sonications with $\mathrm{ZnH}$ and HMT solutions after purification.
4.4 Formation of Tendril Particles. ZnO HPs were coated with 2 bilayers of polyacrylic acid and poly(allyamine hydrochloride) at $\mathrm{pH} 8$ and $\mathrm{M} \mathrm{NaCl}$ in a layer-by-layer method. Each layer consisted of incubating HPs in the polyelectrolyte for $20 \mathrm{~min}$, and then centrifuge washing twice with a $0.1 \mathrm{M} \mathrm{NaCl} \mathrm{pH} 8$ buffer solution and twice with DI water. The polymer layers were then cross-linked using glutaraldehyde (2.5\%). A $4 \mathrm{~mL}$ of sample was incubated with $2 \mathrm{~mL}$ of glutaraldehyde (25\%) and $32 \mathrm{~mL}$ of $1 \mathrm{M}$ boric acid for $2 \mathrm{~h}$. This sample was then washed 3 times with DI water and then placed into an additional acid wash (nitric acid or boric acid) to produce different Zn content TPs (Table Si). TPs were incubated in poly(N-isopropylacrylamide-co-acrylic acid $(1 \mathrm{mg} / \mathrm{mL} ; \mathrm{pH} 8)$ for 20 minutes and then centrifuge washed four times to create PNIPAM TPs. Crosslinking of 1ethyl-3-(3-dimethylaminopropyl)carbodiimide (EDC) TPs was accomplished with $10 \mathrm{mM}$ EDC at $\mathrm{pH} 5.6$ for $2 \mathrm{~h}$ in $0.1 \mathrm{M}$ PBS buffer. Dopamine-modified TPs were created by adsorbing 1 additional (PAA/PAH) bilayer and then were cross-linked with $0.05 \mathrm{M}$ dopamine and 2.5\% glutaraldehyde in o.1M PBS buffer.

4.5 Confocal Microscopy Imaging. For confocal studies, FITC was conjugated to PAH according to a literature procedure. ${ }^{3,58}$ Briefly, $4.5 \mathrm{mg}$ FITC was dissolved in $1 \mathrm{~mL}$ dimethylsulfoxide (DMSO) and combined with $500 \mathrm{mg} \mathrm{PAH}$ dissolved in $6 \mathrm{~mL}$ water. The $\mathrm{pH}$ of the solution was adjusted to 8.4 with $\mathrm{NaOH}$ and then the solution was stirred for $2 \mathrm{~d}$. Dialysis was then performed using a D-tube ${ }^{\mathrm{TM}}$ Dialyzer for 24 $\mathrm{h}$ to remove excess FITC..$^{8}$ PAA/FITC-PAH films were deposited on HPs using LBL assembly as described previously. ${ }^{3}$

4.6 Electron Microscopy Imaging. A $20 \mu \mathrm{L}$ of sample was evaporated on a silicon wafer. . Particles were imaged using a FEI Nova 200 Nanolab SEM and FEI Helios 650 Nanolab SEM/FIB. For dispersion SEM images, particles were imaged at a concentration of $0.1 \mathrm{mg} / \mathrm{mL}$. STEM and TEM images were taken using a Talos F20oX G2, JEOL 3011, and JEOL 2010. EDS was run on samples at a magnification of $65 \mathrm{k} \mathrm{a}$ minimum of 3 times over the spikes of TPs.

4.7 FDTD Simulation of Optical Properties. A model particle was constructed with a core $\mathrm{SiO}_{2}$ sphere (diameter $=1$ $\mu \mathrm{m})$ meta-shell containing a spherical array of $500 \mathrm{ZnO}$ NRs (length $=1463 \mathrm{~nm}$, thickness $=139 \mathrm{~nm}$ ) to approximate the 
experimental construct. The length and thickness were measured using electron microscopy of a HP (minimum of 50 images), which was used for elaboration of TP model geometry. In the numerical calculation of the scattering cross-section, the refractive indices of the tendrils having various $\mathrm{ZnO}$ content were calculated utilizing MaxwellGarnet Medium approximations, where the quantity of $\mathrm{ZnO}$ content are reflected in the volume fraction. As the spectral focus of our investigation lie in the visible wavelength range, where the absorption for the TP components are negligible, extinction coefficients were not taken into consideration in the calculation and the following materials refractive indices were used in the approximation: 1.97 for $\mathrm{ZnO}, 1.53$ for polymer, and 1.46 for $\mathrm{SiO}_{2}$.

4.8 Absorbance Measurements. Absorbance measurements were performed using a Cary 8454 . Measurements were taken every $10 \mathrm{~s}$ for $30 \mathrm{~min}$ at a $275 \mathrm{~nm}$ wavelength in a quartz cuvette. Particles were loaded at 0.5 $\mathrm{mg} / \mathrm{mL}$ in a $3 \mathrm{~mL}$ cuvette. For $\mathrm{pH}$-responsive testing, TPs were measured in o.1 M PBS buffer solution adjusted to $\mathrm{pH}$ 5.6 for acidic conditions and 7.4 for alkaline conditions.

4.9 Molecular Loading of TPs Loading of FITC-Dextran (4k MW) and rhodamine B were carried out in $\mathrm{pH}_{3}$ solution. Encapsulation involved a $\mathrm{pH} 3 \mathrm{TP}$ solution with FITCDextran or rhodamine $\mathrm{B}(\mathrm{1} \mathrm{mM})$ being washed at $\mathrm{pH} 10$ repeatedly to ensure that there was no excess fluorophore in solution, and verified using confocal microscopy imaging. Loading of nile red was done in ethanol $(1 \mathrm{mM})$ with repeated washing with ethanol and then $\mathrm{pH}_{3}$ or $\mathrm{pH}$ solution.

\section{ASSOCIATED CONTENT}

Supporting Information. Additional characterization, dispersion stability and stimuli-responsive results including Figures $\mathrm{S}_{1}-\mathrm{S}_{22}$ and Table S1. This material is available free of charge via the Internet at http://pubs.acs.org.

\section{AUTHOR INFORMATION}

\section{Corresponding Author}

*kotov@umich.edu.

\section{ACKNOWLEDGMENT}

The authors would like to acknowledge funding from the Electric Power Research Institute. Additionally, the authors would like to acknowledge the Michigan center for Materials Characterization and NSF grants DMR-0315633 and DMR0320740 for funding of the microscopes in this work. This work was also supported by NSF 1566460 "Nanospiked Particles for Photocatalysis".

\section{REFERENCES}

(1) Lan, Y.; Caciagli, A.; Guidetti, G.; Yu, Z.; Liu, J.; Johansen, V. E.; Kamp, M.; Abell, C.; Vignolini, S.; Scherman, O. A.; et al. Unexpected Stability of Aqueous Dispersions of Raspberrylike Colloids. Nat. Commun. 2018, 9.

(2) Jiang, W.; Qu, Z.; Kumar, P.; Vecchio, D.; Wang, Y.; Ma, Y.; Bahng, J. H.; Bernardino, K.; Gomes, W. R.; Colombari, F. M.; et al. Emergence of Complexity in Hierarchically Organized Chiral Particles. Science. 2o2o, 368, 642-648.

(3) Montjoy, D. G.; Bahng, J. H.; Eskafi, A.; Hou, H.; Kotov, N. A. Omnidispersible Hedgehog Particles with Multilayer
Coatings for Multiplexed Biosensing. J. Am. Chem. Soc. 2018, 140, 7835-7845.

(4) Bahng, J. H.; Yeom, B.; Wang, Y.; Tung, S. O.; Hoff, J. D.; Kotov, N. Anomalous Dispersions of 'Hedgehog' Particles. Nature 2014, 517, 596-599.

(5) Decher, G. Fuzzy Nanoassemblies: Toward Layered Polymeric Multicomposites. Science 1997, 277, 1232-1237.

(6) Kotov, N. A.; Dékány, I.; Fendler, J. H. Ultrathin Graphite Oxide-Polyelectrolyte Composites Prepared by SelfAssembly: Transition between Conductive and NonConductive States. Adv. Mater. 1996, 8, 637-641.

(7) Stockton, W. B.; Rubner, M. F. Molecular-Level Processing of Conjugated Polymers. 4. Layer-by-Layer Manipulation of Polyaniline via Hydrogen-Bonding Interactions. Macromolecules 1997, 30, 2717-2725.

Sukhorukov, G. B.; Donath, E.; Davis, S.; Lichtenfeld, H.; Caruso, F.; Popov, V. I.; Möhwald, H. Stepwise Polyelectrolyte Assembly on Particle Surfaces: A Novel Approach to Colloid Design. Polym. Adv. Technol. 1998, 9, 759-767.

(9) Lvov, Y.; Yamada, S.; Kunitake, T. Non-Linear Optical Effects in Layer-by-Layer Alternate Films of Polycations and an Azobenzene-Containing Polyanion. Thin Solid Films 1997, 300, 107-112.

(10) Lvov, Y. M.; Lu, Z.; Schenkman, J. B.; Zu, X.; Rusling, J. F. Direct Electrochemistry of Myoglobin and Cytochrome $\mathrm{P}_{450}$ (Cam) in Alternate Layer-by-Layer Films with DNA and Other Polyions. J. Am. Chem. Soc. 1998, 120, 4073-408o.

(11) Xie, X.; Melosh, N. A. Fabrication of Sub-Cell Size "Spiky" Nanoparticles and Their Interfaces with Biological Cells. J. Mater. Chem. B 2015, 3, 5155-516o.

(12) Qin, Y.; Zhou, Y.; Li, J.; Ma, J.; Shi, D.; Chen, J.; Yang, J. Fabrication of Hierarchical Core-Shell Au@ZnO Heteroarchitectures Initiated by Heteroseed Assembly for Photocatalytic Applications. J. Colloid Interface Sci. 2014, 418, 171-177.

(13) Wang, J.; Chen, H. J.; Hang, T.; Yu, Y.; Liu, G.; He, G.; Xiao, S.; Yang, B. ru; Yang, C.; Liu, F.; et al. Physical Activation of Innate Immunity by Spiky Particles. Nat. Nanotechnol. 2018, 13, 1078-1086.

(14) Qiao, L.; Zhao, W.; Qin, Y.; Swihart, M. T. Controlled Growth of a Hierarchical Nickel Carbide "Dandelion" Nanostructure. Angew. Chemie 2o16, 128, 8155-8158.

(15) Yin, B.; Liu, X.; Gao, H.; Fu, T.; Yao, J. Bioinspired and Bristled Microparticles for Ultrasensitive Pressure and Strain Sensors. Nat. Commun. 2018, 9, 1-8.

(16) Deng, D.; Hao, C.; Sen, S.; Xu, C.; Král, P.; Kotov, N. A. Template-Free Hierarchical Self-Assembly of Iron Diselenide Nanoparticles into Mesoscale Hedgehogs. J. Am. Chem. Soc. 2017, 139, 16630-16639.

(17) Kirchner, S. R.; Su, M. N.; Bahng, J. H.; Montjoy, D. G.; Chang, W. S.; Kotov, N. A.; Link, S. Scattering Properties of Individual Hedgehog Particles. J. Phys. Chem. C 2018, 122, 12015-12021.

(18) Hu, X.; Hu, J.; Tian, J.; Ge, Z.; Zhang, G.; Luo, K.; Liu, S. Polyprodrug Amphiphiles: Hierarchical Assemblies for Shape-Regulated Cellular Internalization, Trafficking, and Drug Delivery. J. Am. Chem. Soc. 2o13, 135, 17617-17629.

(19) Niu, Y.; Yu, M.; Hartono, S. B.; Yang, J.; Xu, H.; Zhang, H.; Zhang, J.; Zou, J.; Dexter, A.; Gu, W.; et al. Nanoparticles Mimicking Viral Surface Topography for Enhanced Cellular Delivery. Adv. Mater. 2013, 25, 6233-6237.

(20) Schneider, G.; Decher, G.; Nerambourg, N.; Praho, R.; Werts, M. H. V; Blanchard-Desce, M. Distance-Dependent Fluorescence Quenching on Gold Nanoparticles Ensheathed with Layer-by-Layer Assembled Polyelectrolytes. Nano Lett. 2oo6, 6, 530-536.

(21) Crisp, M. T.; Kotov, N. A. Preparation of Nanoparticle Coatings on Surfaces of Complex Geometry. Nano Lett. 2003, 3, 173-177.

(22) Caruso, F.; Caruso; R.; Möhwald, H. Nanoengineering of 
Inorganic and Hybrid Hollow Spheres by Colloidal Templating. Science (80-. ). 1998, 282, 1111-1114.

(23) Podsiadlo, P.; Kaushik, A. K.; Arruda, E. M.; Waas, A. M.; Shim, B. S.; Xu, J.; Nandivada, H.; Pumplin, B. G.; Lahann, J.; Ramamoorthy, A.; et al. Ultrastrong and Stiff Layered Polymer Nanocomposites. Science 2007, 318, 80-83.

(24) Tong, W.; Gao, C.; Möhwald, H. Manipulating the Properties of Polyelectrolyte Microcapsules by Glutaraldehyde Cross-Linking. Chem. Mater. 2005, 17, 46104616.

(25) Richert, L.; Engler, A. J.; Discher, D. E.; Picart, C. Elasticity of Native and Cross-Linked Polyelectrolyte Multilayer Films. Biomacromolecules 2004, 5, 1908-1916.

(26) Erik, J.; Krishnakumar, A.; Mu, D. Layer-by-Layer Assembly of a Magnetic Nanoparticle Shell on a Thermoresponsive Microgel Core. 2007, 311, 219-223.

(27) Delcea, M.; Möhwald, H.; Skirtach, A. G. StimuliResponsive LbL Capsules and Nanoshells for Drug Delivery. Adv. Drug Deliv. Rev. 2011, 63, 730-747.

(28) Sakr, O. S.; Borchard, G. Encapsulation of Enzymes in Layer-by-Layer (LbL) Structures: Latest Advances and Applications. Biomacromolecules 2013, 14, 2117-2135.

(29) Caruso, F. Nanoengineering of Particle Surfaces. Adv. Mater. 2001, 13, 11-22.

(30) Ferreira, D. C. M.; Mendes, R. K.; Kubota, L. T. Kinetic Studies of HRP Adsorption on Ds-DNA Immobilized on Gold Electrode Surface by EIS and SPR. J. Braz. Chem. Soc. 2010, 21, 1648-1655.

(31) Aroca, R. F.; Goulet, P. J. G.; Dos Santos, D. S.; AlvarezPuebla, R. A.; Oliveira, O. N. Silver Nanowire Layer-byLayer Films as Substrates for Surface-Enhanced Raman Scattering. Anal. Chem. 2005, 77, 378-382.

(32) Correa, S.; Boehnke, N.; Deiss-Yehiely, E.; Hammond, P. T. Solution Conditions Tune and Optimize Loading of Therapeutic Polyelectrolytes into Layer-by-Layer Functionalized Liposomes. ACS Nano 2019, 13, 5623-5634.

(33) Ochs, C. J.; Such, G. K.; Yan, Y.; Van Koeverden, M. P.; Caruso, F. Biodegradable Click Capsules with Engineered Drug-Loaded Multilayers. ACS Nano 2010, 4, 1653-1663.

(34) Richardson, J. J.; Choy, M. Y.; Guo, J.; Liang, K.; Alt, K.; Ping, Y.; Cui, J.; Law, L. S.; Hagemeyer, C. E.; Caruso, F. Polymer Capsules for Plaque-Targeted In Vivo Delivery. Adv. Mater. 2016, 28, 7703-7707.

(35) Wang, F.; Bazan, G. C. Aggregation-Mediated Optical Properties of PH-Responsive Anionic Conjugated Polyelectrolytes. J. Am. Chem. Soc. 2oo6, 128, 15786-15792.

(36) Borges, J.; Rodrigues, L. C.; Reis, R. L.; Mano, J. F. Layer-byLayer Assembly of Light-Responsive Polymeric Multilayer Systems. Adv. Funct. Mater. 2014, 24, 5624-5648.

(37) Bunyakan, C.; Hunkeler, D. Precipitation Polymerization of Acrylic Acid in Toluene . I : Synthesis, Characterization and Kinetics. 1999, 40, 6213-6224.

(38) Sukhorukov, G. B.; Donath, E.; Lichtenfeld, H.; Knippel, E.; Knippel, M.; Budde, A.; Möhwald, H. Layer-by-Layer Self Assembly of Polyelectrolytes on Colloidal Particles. Colloids Surfaces A Physicochem. Eng. Asp. 1998, 137, 253-266.

(39) Soomro, M. Y.; Hussain, I.; Bano, N.; Broitman, E.; Nur, O.; Willander, M. Nanoscale Elastic Modulus of Single Horizontal $\mathrm{ZnO}$ Nanorod Using Nanoindentation Experiment. Nanoscale Res. Lett. 2012, 7, 146.

(40) Shao, L.; Lutkenhaus, J. L. Thermochemical Properties of Free-Standing Electrostatic Layer-by-Layer Assemblies Containing Poly(Allylamine Hydrochloride) and Poly(Acrylic Acid). Soft Matter 2o1o, 6, 3363-3369.

(41) Angelatos, A. S.; Radt, B.; Caruso, F. Light-Responsive Polyelectrolyte/Gold Nanoparticle Microcapsules. J. Phys. Chem. B 2005, 109, 3071-3076.

(42) Donath, E.; Sukhorukov, G. B.; Caruso, F.; Davis, S. A.;
Möhwald, H. Novel Hollow Polymer Shells by ColloidTemplated Assembly of Polyelectrolytes. Angew. Chemie Int. Ed. 1998, 2201-2205.

(43) Sukhorukov, G. B.; Antipov, A. A.; Voigt, A.; Donath, E.; Möhwald, H. PH-Controlled Macromolecule Encapsulation in and Release from Polyelectrolyte Multilayer Nanocapsules. Macromol. Rapid Commun. 2oo1, 22, 44-46.

(44) Caruso, F.; Möhwald, H. Protein Multilayer Formation on Colloids through a Stepwise Self- Assembly Technique. J. Am. Chem. Soc. 1999, 121, 6039-6046.

(45) Tong, W.; Gao, C.; Möhwald, H. Manipulating the Properties of Polyelectrolyte Microcapsules by Glutaraldehyde Cross-Linking. Chem. Mater. 2005, 17, 46104616.

(46) Shchukin, D. G.; Lamaka, S. V.; Yasakau, K. A.; Zheludkevich, M. L.; Ferreira, M. G. S.; Möhwald, H. Active Anticorrosion Coatings with Halloysite Nanocontainers. J. Phys. Chem. C 2008, 112, 958-964.

(47) Szarpak, A.; Cui, D.; Dubreuil, F.; De Geest, B. G.; De Cock, L. J.; Picart, C.; Auzély-Velty, R. Designing Hyaluronic AcidBased Layer-by-Layer Capsules as a Carrier for Intracellular Drug Delivery. Biomacromolecules 2010, 11, 713-720.

(48) Mueller, M.; Tebbe, M.; Andreeva, D. V.; Karg, M.; Alvarez Puebla, R. A.; Pazos Perez, N.; Fery, A. Large-Area Organization of PNIPAM-Coated Nanostars as SERS Platforms for Polycyclic Aromatic Hydrocarbons Sensing in Gas Phase. Langmuir 2012, 28, 9168-9173.

(49) J. E., C.; M., Y. Y.; T., A.; Y., S.; T., O.; Chung, J. E.; Yokoyama, M.; Yamato, M.; Aoyagi, T.; Sakurai, Y.; et al. Thermo-Responsive Drug Delivery from Polymeric Micelles Constructed Using Block Copolymers of Poly(NIsopropylacrylamide) and Poly(Butylmethacrylate). J. Control. Release 1999, 62, 115-127.

(5o) Schild, H. G. Poly(N-Isopropylacrylamide): Experiment, Theory and Application. Prog. Polym. Sci. 1992, 17, 163-249.

(51) Min, Y.; Hammond, P. T. Catechol-Modified Polyions in Layer-by-Layer Assembly to Enhance Stability and Sustain Release of Biomolecules: A Bioinspired Approach. Chem. Mater. 2011, 23, 5349-5357.

(52) Xia, L. W.; Xie, R.; Ju, X. J.; Wang, W.; Chen, Q.; Chu, L. Y. Nano-Structured Smart Hydrogels with Rapid Response and High Elasticity. Nat. Commun. 2013, 4, 1-11.

(53) Kim, S. K.; Chang, H.; Cho, K.; Kil, D. S.; Cho, S. W.; Jang, H. D.; Choi, J. W.; Choi, J. Enhanced Photocatalytic Property of Nanoporous $\mathrm{TiO}_{2} / \mathrm{SiO}_{2}$ Micro-Particles Prepared by Aerosol Assisted Co-Assembly of Nanoparticles. Mater. Lett. 2011, 65, 3330-3332.

(54) Lee, H.; Scherer, N. F.; Messersmith, P. B. Single-Molecule Mechanics of Mussel Adhesion. Proc. Natl. Acad. Sci. 20o6, 103, 12999-13003.

(55) Stöber, W.; Fink, A.; Bohn, E. Controlled Growth of Monodisperse Silica Spheres in the Micron Size Range. J. Colloid Interface Sci. 1968, 26, 62-69.

(56) Yu, B.; Cong, H.; Xue, L.; Tian, C.; Xu, X.; Peng, Q.; Yang, S. Synthesis and Modification of Monodisperse Silica Microspheres for UPLC Separation of C6o and C7o. Anal. Methods 2016, 8, 919-924.

(57) Greasley, S. L.; Page, S. J.; Sirovica, S.; Chen, S.; Martin, R. A.; Riveiro, A.; Hanna, J. V.; Porter, A. E.; Jones, J. R. Controlling Particle Size in the Stöber Process and Incorporation of Calcium. J. Colloid Interface Sci. 2016, 469, 213-223.

(58) Begum, G.; Singh, S.; Rangaraj, N.; Srinivas, G.; Rana, R. K. Cellular Permeation with Nuclear Infiltration Capability of Biomimetically Synthesised Fluorescent Monodisperse Mesoporous Silica Nanospheres in HeLa and Human Stem Cells. J. Mater. Chem. 2010, 20, 8563. 